\title{
Unique representation of an inverse-kinetic theory for incompressible Newtonian fluids
}

\author{
M. Tessarotto ${ }^{a}$ and M. Ellero ${ }^{b}$ \\ ${ }^{a}$ Department of Mathematics and Informatics, University of Trieste, Trieste, Italy \\ and Consortium for Magnetofluid Dynamics, Trieste, Italy \\ ${ }^{\mathrm{b}}$ School of Aerospace, Mechanical and Mechatronic Engineering, The University of \\ Sydney, NSW 2006, Australia
}

\begin{abstract}
Fundamental aspects of inverse kinetic theories for the incompressible Navier-Stokes equations [Ellero and Tessarotto, 2004, 2005] include the possibility of defining uniquely the kinetic equation underlying such models and furthermore, the construction of a kinetic theory implying also the energy equation. The latter condition is consistent with the requirement that fluid fields result classical solutions of the fluid equations. These issues appear of potential relevance both from the mathematical viewpoint and for the physical interpretation of the theory. Purpose of this work is to prove that under suitable prescriptions the inverse kinetic theory can be determined to satisfy such requirements.
\end{abstract}

PACS: 47.27.Ak, 47.27.Eq, 47.27.Jv

Key words:

Incompressible Navier-Stokes equations: kinetic theory; Fokker-Planck equation.

\section{Introduction}

An aspect of fluid dynamics is represented by the class of so-called inverse problems, involving the search of model kinetic theories able to yield a prescribed complete set of fluid equations advancing in time a suitable set of fluid fields. This is achieved by means of suitable velocity-moments of an appropriate kinetic distribution function $f(\mathbf{r}, \mathbf{v}, t)$. Among such model theories, special

$\overline{1}$ Corresponding author: email: marco.ellero@cmfd.univ.trieste.it 
relevance pertains to those in which the state of isothermal incompressible fluids is described self-consistently by suitable fluid fields to be considered as classical (i.e., strong) solutions of the corresponding fluid equations (regularity assumption). In this case the relevant fluid fields are the mass density, fluid velocity and fluid pressure $\{\rho, \mathbf{V}, p\}$ which are required to be classical solutions of the so-called incompressible Navier-Stokes equations (INSE)

$$
\begin{aligned}
\frac{\partial}{\partial t} \rho+\nabla \cdot(\rho \mathbf{V}) & =0, \\
\rho \frac{D}{D t} \mathbf{V}+\nabla p+\mathbf{f}-\mu \nabla^{2} \mathbf{V} & =\mathbf{0}, \\
\nabla \cdot \mathbf{V} & =0, \\
\rho(\mathbf{r}, t) & >0, \\
p(\mathbf{r}, t) & \geq 0, \\
\rho(\mathbf{r}, t) & =\rho_{o}>0 .
\end{aligned}
$$

The first three equations (1),(2) and (3), denoting respectively the continuity, Navier-Stokes and isochoricity equations, are assumed to be satisfied in the open set $\Omega \subseteq \mathbb{R}^{3}$ (fluid domain) and in a possibly bounded time interval $I \subset \mathbb{R}$, while the last three inequalities, (4)-(6) apply also in the closure of the fluid domain $\bar{\Omega} \equiv \Omega \cup \delta \Omega$. Here the notation is standard. Hence $\frac{D}{D t}=\frac{\partial}{\partial t}+\mathbf{V} \cdot \nabla$ is the convective derivative, $\mathbf{f}(\mathbf{r}, t)$ denotes the volume force density acting on the fluid element and $\mu \equiv \nu \rho_{o}>0$ is the constant fluid viscosity, $\nu=\mu / \rho_{o}$ being the related kinematic viscosity. It is assumed that the fluid fields and $\mathbf{f}(\mathbf{r}, t)$ are suitably smooth to belong to the functional setting

$$
\left\{\begin{array}{l}
\mathbf{V}(\mathbf{r}, t), p(\mathbf{r}, t), \mathbf{f}(\mathbf{r}, \mathbf{v}, t) \in C^{(0)}(\bar{\Omega} \times I) \\
\mathbf{V}(\mathbf{r}, t), p(\mathbf{r}, t) \in C^{(2,1)}(\Omega \times I) \\
\mathbf{f}(\mathbf{r}, t) \in C^{(1,0)}(\Omega \times I)
\end{array}\right.
$$

[where $\left.C^{(i, j)}(\Omega \times I) \equiv C^{(i,)}(\Omega) \times C^{(j)} I\right)$, with $i, j \in \mathbb{N}$ ]. Hence, $\{\rho, \mathbf{V}, p\}$ are classical solutions of INSE so that the energy equation, obtained by taking the scalar product of the Navier-Stokes equation by the fluid velocity $\mathbf{V}$, holds identically in the same domain

$$
\frac{D}{D t} \frac{V^{2}}{2}+\frac{1}{\rho_{o}} \mathbf{V} \cdot \nabla p+\frac{1}{\rho_{o}} \mathbf{V} \cdot \mathbf{f}-\nu \mathbf{V} \cdot \nabla^{2} \mathbf{V}=0 .
$$

The set of equations (1)-(6), together with (8), will be denoted in the sequel as extended INSE.

An inverse kinetic theory for INSE is therefore represented by a kinetic equation of the form

$$
L(\mathbf{F}) f=0
$$


$f(\mathbf{x}, t)$ being a kinetic distribution function, defined in the extended phase space $\Gamma \times I$, where $\Gamma=\Omega \times U$ (with $\Gamma$ and $U$ the phase and velocity spaces), $\bar{\Gamma}=\bar{\Omega} \times U$ its closure, while $\mathbf{x} \equiv(\mathbf{r}, \mathbf{v}) \in \Gamma$ and $\mathbf{v} \in U \equiv \mathbb{R}^{3}$ denote respectively the state and velocity variables. The distribution $f(\mathbf{x}, t)$ is assumed suitably regular (i.e., suitably smooth in $\Gamma \times I$ and summable in $\Gamma$ ) and to obey appropriate initial and boundary conditions. $L(\mathbf{F})$ is an appropriate operator (to be denoted as Vlasov streaming operator). This is defined in such a way that appropriate velocity moments of (9), are assumed to exist, yield INSE. In particular, $L(\mathbf{F})$ can be defined in such a way to allow that the inverse kinetic equation (9) admits, as a particular solution, the local Maxwellian distribution

$$
f_{M}\left(\mathbf{x}, t ; \mathbf{V}, p_{1}\right)=\frac{\rho_{0}^{5 / 2}}{(2 \pi)^{\frac{3}{2}} p_{1}^{\frac{3}{2}}} \exp \left\{-X^{2}\right\}
$$

Here, the notation is standard [3] , thus

$$
\begin{aligned}
X^{2} & =\frac{u^{2}}{v_{t h}{ }^{2}}, \\
v_{t h}^{2} & =2 p_{1} / \rho_{o},
\end{aligned}
$$

$p_{1}$ being the kinetic pressure. Desirable features of the inverse kinetic theory involve the requirement that, under suitable assumptions, the functional form of the relevant inverse kinetic equation, yielding the INSE equations, be uniquely defined. In addition, it might be convenient to impose also the validity of additional fluid equations, such for example the energy equation (8). In fact, it is well known that the energy equation is not satisfied by weak solutions of INSE and, as a consequence, also by certain numerical schemes. Therefore, the validity of the inverse kinetic equation yields a necessary condition for the existence of classical solutions for INSE.

Concerning the first point, the prescription of uniqueness on the kinetic equation has to be intended in a suitably meaningful sense, i.e., to hold under the requirement that the relevant set of fluid equations are fulfilled identically by the fluid fields in the extended domain $\Omega \times I$. This means that arbitrary contributions in the kinetic equation, which vanish identically under a such an hypothesis, can be included in the same kinetic equation. Consistently with the previous regularity assumption, here we intend to consider, in particular, the requirement that the inverse kinetic equation yields also the energy equation (8).

In a previous work [2, 3], an explicit solution to INSE has been discovered based on a continuous inverse kinetic theory, adopting a Vlasov differential kinetic equation defined by a suitable streaming operator $L$. Basic feature of the kinetic equation is that, besides yielding INSE as moment equations, it 
allows as particular solution local kinetic Maxwellian equilibria for arbitrary fluid fluids $\left\{\rho_{o}, \mathbf{V}, p\right\}$ which belong to the above functional setting (7). However, as pointed out in [3], the inverse kinetic equation defined in this way results parameter-dependent and hence non-unique, even in the case of local Maxwellian kinetic equilibria. This non-uniqueness feature may result as a potentially undesirable feature of the mathematical model, since it prevents the possible physical interpretation of the theory (in particular, of the meanfield force $\mathbf{F}$ ) and may result inconvenient from the numerical viewpoint since $|\alpha|$ may be chosen, for example, arbitrarily large. Hence it would be highly desirable to eliminate it from the theory.

The purpose of this paper is twofold.

First we intend to prove that under suitable prescriptions the inverse kinetic equation can be cast in an unique form, thus eliminating possible parameterdependences in the relevant streaming operator $[L(\mathbf{F})]$.This is achieved by analyzing the form of the streaming operator for particular solutions (local Maxwellian kinetic equilibria). In this case the the kinetic equation can be cast uniquely in an equivalent symmetrized form represented by a Vlasov streaming operator.

As further development of the theory, it is shown that the streaming operator can be suitably modified in such a way that the inverse kinetic equation yields the extended INSE equations, i.e., besides the incompressible Navier-Stokes equations also the energy equation. In particular we intend to prove that the mean-field force $\mathbf{F}$ can be uniquely defined in such a way that both kinetic equilibrium and moment equations yield uniquely such equations.

The scheme of the presentation is as follows. In Sec.2 the inverse kinetic equation developed in [3] is recalled and the non-uniqueness feature of the meanfield force $\mathbf{F}$ is analyzed. In Sec.3 an equivalent representation of the streaming operator is introduced which permits to define uniquely F. As a result, a uniqueness theorem is obtained for the streaming operator $L(\mathbf{F})$. Finally in Sec. 4 an extension of the inverse kinetic theory is presented which provides a solution also for the energy equation, besides the incompressible Navier-Stokes equations. The formulation of the inverse kinetic equation for the extended set of fluid equations is obtained by a suitable redefinition of the mean-field force $\mathbf{F}$. Also in such a case the vector field $\mathbf{F}$ is proven to be unique.

\section{Non-uniqueness of the streaming operator}

Goal of this Section is to investigate the kinetic equation developed in [3] to analyze its non-uniqueness features. We start recalling the inverse kinetic 
equation, which is assumed to be of the form (9) or $L(\mathbf{F}) \widehat{f}=0, \widehat{f}(\mathbf{x}, t)$ being the normalized kinetic distribution function associated to the kinetic distribution function $f(\mathbf{x}, t)$,

$$
\widehat{f}(\mathbf{x}, t) \equiv f(\mathbf{x}, t) / \rho_{o} .
$$

In particular, the streaming operator $L$ is assumed to be realized by a differential operator of the form

$$
\begin{aligned}
& L(\mathbf{F})=\frac{\partial}{\partial t}+\mathbf{v} \cdot \frac{\partial}{\partial \mathbf{r}}+ \\
& +\frac{\partial}{\partial \mathbf{v}} \cdot\{\mathbf{F}\}
\end{aligned}
$$

and $\mathbf{F}(\mathbf{r}, \mathbf{v}, t ; f)$ an appropriate vector field (mean-field force) defined by Eq.(42) (see Appendix A) in terms of vector fields $\mathbf{F}_{0}$ and $\mathbf{F}_{1}$. As a consequence, both $\mathbf{F}_{0}$ and $\mathbf{F}_{1}$ are functionally dependent on the form of the kinetic distribution function $f(\mathbf{x}, t)$. In particular, requiring that $\mathbf{F}$ depends on the minimal number of velocity moments (see below), it is defined by Eqs. (42),(43) and (44), given in Appendix A. Supplemented with suitable initial and boundary conditions and subject to suitable smoothness assumptions for the kinetic distribution function $f(\mathbf{x}, t)$, several important consequences follow [3]:

- the fluid fields $\left\{\rho_{o}, \mathbf{V}, p\right\}$ can be identified in the whole fluid domain $\Omega$ with suitable velocity moments (which are assumed to exist) of the kinetic distribution function $f(\mathbf{x}, t)$ [or equivalent $\widehat{f}(\mathbf{x}, t)$ ], of the form

$$
M_{G}(r, t)=\int d^{3} v G(\mathbf{x}, t) f(\mathbf{x}, t)
$$

where $G(\mathbf{x}, t)=1, \mathbf{v}, E \equiv \frac{1}{3} u^{2}, \mathbf{v} E, \mathbf{u u}$, and

$$
\mathbf{u} \equiv \mathbf{v}-\mathbf{V}(\mathbf{r}, t)
$$

is the relative velocity. Thus, we require respectively

$$
\begin{gathered}
\rho_{o}=\int d^{3} v f(\mathbf{x}, t), \\
\mathbf{V}(\mathbf{r}, t)=\frac{1}{\rho} \int d^{3} v \mathbf{v} f(\mathbf{x}, t), \\
p(\mathbf{r}, t)=p_{1}(\mathbf{r}, t)-P_{o},
\end{gathered}
$$

$p_{1}(\mathbf{r}, t)$ being the scalar kinetic pressure, i.e.,

$$
p_{1}(\mathbf{r}, t)=\int d \mathbf{v} \frac{u^{2}}{3} f(\mathbf{x}, t),
$$

Requiring, $\nabla p(\mathbf{r}, t)=\nabla p_{1}(\mathbf{r}, t)$ and $p_{1}(\mathbf{r}, t)$ strictly positive, it follows that $P_{o}$ is an arbitrary strictly positive function of time, to be defined so that the 
physical realizability condition $p(\mathbf{r}, t) \geq 0$ is satisfied everywhere in $\bar{\Omega} \times I$ $(I \subseteq \mathbb{R}$ being generally a finite time interval);

- $\left\{\rho_{o}, \mathbf{V}, p\right\}$ are advanced in time by means of the inverse kinetic equation Eq.(9);

- By appropriate choice of the mean-field force $\mathbf{F}$, the moment equations can be proven to satisfy identically INSE, and in particular the Poisson equation for the fluid pressure, as well the appropriate initial and boundary conditions (see Ref.[3]);

- The mean-field force $\mathbf{F}$ results, by construction, function only of the velocity moments (37), i.e., $\left\{\rho_{o}, \mathbf{V}, p_{1}, \mathbf{Q}, \underline{\underline{\mathbf{\Pi}}}\right\}$, to be denoted as extended fluid

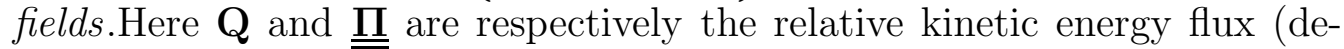
fined in the reference frame locally at rest with respect to the fluid) and the pressure tensor

$$
\begin{aligned}
& \mathbf{Q}=\int d^{3} v \mathbf{u} \frac{u^{2}}{3} f, \\
& \underline{\underline{\boldsymbol{\Pi}}}=\int d^{3} v \mathbf{u} \mathbf{u} f
\end{aligned}
$$

- The Maxwellian kinetic distribution function $f_{M}$, defined by the equation (10), results a particular solution of the inverse kinetic equation (9) if and only if $\{\rho, \mathbf{V}, p\}$ satisfy INSE.

Let us now prove that the inverse kinetic equation defined above (9) is nonunique, even in the particular case of local Maxwellian kinetic equilibria, due to the non-uniqueness in the definition of the mean-field force $\mathbf{F}$ and the streaming operator $L(\mathbf{F})$. In fact, let us introduce the parameter-dependent vector field $\mathbf{F}(\alpha)$

$$
\mathbf{F}(\alpha)=\mathbf{F}+\alpha \mathbf{u} \cdot \nabla \mathbf{V}-\alpha \nabla \mathbf{V} \cdot \mathbf{u} \equiv \mathbf{F}_{0}(\alpha)+\mathbf{F}_{1}
$$

where $\mathbf{F} \equiv \mathbf{F}(\alpha=0), \alpha \in \mathbb{R}$ is arbitrary and we have denoted

$$
\begin{aligned}
& \mathbf{F}_{0}(\alpha)=\mathbf{F}_{0}-\alpha \Delta \mathbf{F}_{0} \equiv \mathbf{F}_{0 a}+\Delta_{1} \mathbf{F}_{0}(\alpha) \\
& \Delta \mathbf{F}_{0} \equiv \mathbf{u} \cdot \nabla \mathbf{V}-\nabla \mathbf{V} \cdot \mathbf{u} \\
& \Delta_{1} \mathbf{F}_{0}(\alpha) \equiv(1+\alpha) \mathbf{u} \cdot \nabla \mathbf{V}-\alpha \nabla \mathbf{V} \cdot \mathbf{u}
\end{aligned}
$$

where $\mathbf{F}_{0}$ and $\mathbf{F}_{1}$ given by Eqs.(43),(44). Furthermore, here we have introduced also the quantity $\Delta_{1} \mathbf{F}_{0}(\alpha)$ to denote the parameter-dependent part of $\mathbf{F}_{0}(\alpha)$. In fact, it is immediate to prove the following elementary results:

a) for arbitrary $\alpha \in \mathbb{R}$, the local Maxwellian distribution (10) $f_{M}$ is a particular solution of the inverse kinetic equation (9) if and only if the incompressible N-S equations are satisfied;

b) for arbitrary $\alpha$ in $\mathbb{R}$, the moment equations stemming from the kinetic equation (9) coincide with the incompressible N-S equations; 
c) the parameter $\alpha$ results manifestly functionally independent of the kinetic distribution function $f(\mathbf{x}, t)$.

The obvious consequence is that the functional form of the vector field $\mathbf{F}_{0}$, and consequently $\mathbf{F}$, which characterizes the inverse kinetic equation (9) is not unique. The non-uniqueness in the contribution $\mathbf{F}_{0}(\alpha)$ is carried by the term $\alpha \Delta \mathbf{F}_{0}$ which does not vanish even if the fluid fields are required to satisfy identically INSE in the set $\Omega \times I$.

We intend to show in the sequel that the value of the parameter $\alpha$ can actually be uniquely defined by a suitable prescription on the streaming operator (14) and the related mean-field force.

\section{A unique representation}

To resolve the non-uniqueness feature of the functional form of the streaming operator (14), due to this parameter dependence, let us now consider again the inverse kinetic equation (9). We intend to prove that the mean-field force $\mathbf{F}$, and in particular the vector field $\mathbf{F}_{0}(\alpha)$, can be given an unique representation in terms of a suitable set of fluid fields $\left\{\rho_{o}, \mathbf{V}, p_{1}, \mathbf{Q}, \underline{\underline{\Pi}}\right\}$ defined by Eqs. (18)-(21) and (22),(23), by introducing a symmetrization condition on the mean field force $\mathbf{F}_{0}(\alpha)$. To reach this conclusion it is actually sufficient to impose that the kinetic energy flux equation results parameter-independent and suitably defined. Thus, let us consider the moment equation which corresponds the kinetic energy flux density $G(\mathbf{x}, t)=\mathbf{v} \frac{u^{2}}{3}$. Requiring that $f(\mathbf{x}, t)$ is an arbitrary particular solution of the inverse kinetic equation (not necessarily Maxwellian) for which the corresponding moment $\mathbf{q}=\int d^{3} v \mathbf{v} \frac{u^{2}}{3} f$ (kinetic energy flux vector) does not vanish identically, the related moment equation takes the form

$$
\begin{aligned}
& \frac{\partial}{\partial t} \int d \mathbf{v} G(\mathbf{x}, t) f+\nabla \cdot \int d \mathbf{v} \mathbf{v} G(\mathbf{x}, t) f- \\
& -\int d \mathbf{v}\left[\mathbf{F}_{0 a}+\Delta_{1} \mathbf{F}_{0}(\alpha)+\mathbf{F}_{1}\right] \cdot \frac{\partial G(\mathbf{x}, t)}{\partial \mathbf{v}} f- \\
& -\int d \mathbf{v} f\left[\frac{\partial}{\partial t} G(\mathbf{x}, t)+\mathbf{v} \cdot \nabla G(\mathbf{x}, t)\right]=0 .
\end{aligned}
$$

Introducing the velocity moments $p_{2}=\int d \mathbf{v} \frac{u^{4}}{3} f, \underline{\underline{\mathbf{P}}}=\int d \mathbf{v u u} \frac{u^{2}}{3} f$ and $\underline{\underline{\mathbf{T}}}=$ $\int d \mathbf{v u u u} f$, the kinetic energy flux equation becomes therefore 


$$
\begin{aligned}
& \frac{\partial}{\partial t} \mathbf{q}+\nabla \cdot \underline{\underline{\mathbf{P}}}+\mathbf{V} \cdot\left[\underline{\underline{\mathbf{P}}}+\mathbf{2} \mathbf{V Q}+\mathbf{V} \mathbf{V} p_{1}\right]+ \\
+ & {\left[\frac{1}{\rho_{o}} \mathbf{f}-\nu \nabla^{2} \mathbf{V}\right] \cdot\left[\underline{\underline{\mathbf{1}}} p_{1}+\frac{2}{3} \underline{\underline{\mathbf{I}}}\right]-} \\
- & {\left[\frac{3}{2} \mathbf{Q}+\mathbf{V} p_{1}\right]\left\{\frac{D}{D t} \ln p_{1}+\frac{1}{p_{1}} \nabla \cdot \mathbf{Q}-\frac{1}{p_{1}^{2}}[\nabla \cdot \underline{\underline{\mathbf{\Pi}}}] \cdot \mathbf{Q}\right\}-} \\
- & \frac{v_{t h}^{2}}{2 p_{1}} \nabla \cdot \underline{\underline{\boldsymbol{\Pi}}} \cdot\left\{\underline{\underline{\mathbf{1}}}\left[\frac{p_{2}}{v_{t h}^{2}}-\frac{3}{2} p_{1}\right]+[\mathbf{Q V}+\underline{\underline{\mathbf{P}}}] \frac{1}{v_{t h}^{2}}-\frac{3}{2} \underline{\underline{\mathbf{\Pi}}}\right\}- \\
- & (1+\alpha) \mathbf{Q} \cdot \nabla \mathbf{V}+\alpha \nabla \mathbf{V} \cdot \mathbf{Q}+\frac{\mathbf{2}}{\mathbf{3}} \nabla \mathbf{V}:(\underline{\underline{\underline{\mathbf{T}}}}+\underline{\underline{\boldsymbol{\Pi}}} \mathbf{V})+ \\
+ & \frac{2}{3} \frac{\partial \mathbf{V}}{\partial t} \cdot \underline{\underline{\mathbf{\Pi}}}+\frac{2}{3} \mathbf{V} \cdot \nabla \mathbf{V} \cdot \underline{\underline{\mathbf{\Pi}}}+\frac{2}{3} \nabla \mathbf{V}: \underline{\underline{\mathbf{T}}}=\mathbf{0} .
\end{aligned}
$$

Unlike the lower-order moment equations (obtained for $G(\mathbf{x}, t)=1, \mathbf{v}, u^{2} / 3$ ), the kinetic energy flux equation contains contributions which depend linearly on the undetermined parameter $\alpha$. These terms, proportional to the velocity gradient $\nabla \mathbf{V}$, yield generally non-vanishing contributions to the rate-of-change of $\mathbf{q}$. The sum of all such terms, which are carried respectively by $\Delta_{1} \mathbf{F}_{0}(\alpha)$, $\mathbf{v} \cdot \nabla G(\mathbf{x}, t)$ and the convective term $\mathbf{V} \cdot \nabla \mathbf{Q}$, reads

$$
\mathbf{M}_{\alpha}(f) \equiv 2 \nabla \cdot(\mathbf{V Q})-(1+\alpha) \mathbf{Q} \cdot \nabla \mathbf{V}+\alpha \nabla \mathbf{V} \cdot \mathbf{Q}
$$

which is the contribution to the rate-of-change of $\mathbf{q}$ which results proportional both to $\mathbf{Q}$ (the relative kinetic energy flux) and the velocity gradient (either $\nabla \mathbf{V}$ or $\mathbf{V} \cdot \nabla)$. In order to eliminate the indeterminacy of $\alpha$, since $\alpha$ cannot depend on the kinetic distribution function $f$, a possible choice is provided by the assumption that $\mathbf{M}_{\alpha}(f)$ takes the symmetrized form

$$
\mathbf{M}_{\alpha}(f)=2 \nabla \cdot(\mathbf{V Q})-\frac{1}{2} \nabla \mathbf{V} \cdot \mathbf{Q}+\frac{1}{2} \mathbf{Q} \cdot \nabla \mathbf{V}
$$

which manifestly implies $\alpha=1 / 2$. Notice that the symmetrization condition can also be viewed as a constitutive equation for the rate-of-change of the kinetic energy flux vector. In this sense, it is analogous to similar symmetrized constitutive equations adopted in customary approaches to extended thermodynamics [11]. On the other hand, Eq.(31) implies $\mathbf{M}_{\alpha}(f)=\frac{1}{2} \mathbf{Q} \times \xi$, $\xi=\nabla \times \mathbf{V}$ being the vorticity field. Thus, $\mathbf{M}_{\alpha}(f)$ can also be interpreted as the rate-of-change of the kinetic energy flux vector $\mathbf{Q}$ produced by vorticity field $\xi$. From Eq.(31) it follows that $\mathbf{F}_{0}(\alpha)$ reads

$$
\begin{aligned}
& \mathbf{F}_{0}\left(\alpha=\frac{1}{2}\right)=-\frac{1}{\rho_{o}} \mathbf{f}+ \\
& +\frac{1}{2}(\mathbf{u} \cdot \nabla \mathbf{V}+\nabla \mathbf{V} \cdot \mathbf{u})+\nu \nabla^{2} \mathbf{V} .
\end{aligned}
$$


As a consequence, the functional form of the streaming operator results uniquely determined. Finally, for completeness, we notice that the same representation for $\mathbf{F}_{0}(\alpha)$ can also be obtained adopting the viewpoint described in Appendix B. In fact, since $\alpha$ is functionally independent of the kinetic distribution function $f(\mathbf{x}, t)$, it can also be defined in such a way to satisfy a suitable symmetry condition in velocity-space, which holds in the particular case $f(\mathbf{x}, t)=f_{M}(\mathbf{x}, t)$. This is can be realized by requiring that the Vlasov streaming operator $L(\mathbf{F})$ coincides in such a case with a suitable Fokker-Planck operator with velocity-independent Fokker-Planck coefficients (see Appendix B).

Finally, it is interesting to point out that the Vlasov streaming operator $L(\mathbf{F})$ defined in terms of $\mathbf{F}_{0}\left(\alpha=\frac{1}{2}\right)$ results by construction Markovian. Hence, the position (32) does not conflict with the Pawula theorem [7, 8] which yields a sufficient condition for the positivity of the kinetic distribution function $f$. The condition of positivity for the kinetic distribution function satisfying the inverse kinetic equation (9) which corresponds to the definition (32) for $\mathbf{F}_{0}(\alpha)$ has been investigated elsewhere [4]. In particular by assuming that $f$ results initially strictly positive and suitably smooth, one can prove that $f$ satisfies an H-theorem both for Maxwellian and non-Maxwellian distributions functions.

As a result of the previous considerations, it is possible to establish the following uniqueness theorem:

\section{THEOREM 1 - Uniqueness of the Vlasov streaming operator $L(\mathbf{F})$}

Let us assume that:

1) the fluid fields $\{\rho, \mathbf{V}, p\}$ and volume force density $\mathbf{f}(\mathbf{r}, \mathbf{V}, t)$ belong to the functional setting ( 7$)$;

2) the operator $L(\mathbf{F})$, defining the inverse kinetic equation (9), has the form of the Vlasov streaming operator (14);

3) the solution, $f(\mathbf{x}, t)$, of the inverse kinetic equation (9) exists, results suitably smooth in $\Gamma \times I$ and its velocity moments $\left\{\rho_{o}, \mathbf{V}, p_{1}, \mathbf{Q}, \underline{\underline{\Pi}}\right\}$ define the fluid fields $\left\{\rho_{o}, \mathbf{V}, p\right\}$ which are classical solutions of INSE, together with Dirichlet boundary conditions and initial conditions. In addition, the inverse kinetic equation admits, as particular solution, the local Maxwellian distribution (10);

4) the mean-field force $\mathbf{F}(\alpha)$ is a function only of the extended fluid fields $\left\{\rho_{o}, \mathbf{V}, p_{1}, \mathbf{Q}, \underline{\underline{\boldsymbol{\Pi}}}\right\}$, while the parameter $\alpha$ does not depend functionally on $f(\mathbf{x}, t)$;

5) the vector field $\Delta_{1} F_{0}(\alpha)$ satisfies the the symmetry condition (31).

Then it follows that the mean-field force $\mathbf{F}$ in the inverse kinetic equation (9) 
is uniquely defined in terms of

$$
\mathbf{F}=\mathbf{F}_{0}+\mathbf{F}_{1}
$$

where the vector fields $\mathbf{F}_{0}$ and $\mathbf{F}_{1}$ are given by Eqs. (32) and (44).

\section{PROOF}

Let us consider first the case in which the distribution function $f(\mathbf{x}, t)$ coincides with the local Maxwellian distribution $f_{M}(10)$. In this case by definition the moments $\mathbf{Q}, \underline{\underline{\Pi}}$ vanish identically while, by construction the mean mean-field force is given by $\mathbf{F}(\alpha)$ [see Eq.()], $\alpha \in \mathbb{R}$ being an arbitrary parameter.

Let us now assume that $f(\mathbf{x}, t)$ is non-Maxwellian and that its moment $\mathbf{M}_{\alpha}(f)$ defined by Eq.(30) is non-vanishing. In this case the uniqueness of $\mathbf{F}$ follows from assumptions 4 and 5. In particular the parameter $\alpha$ is uniquely determined by the symmetry condition (31) in the moment $\mathbf{M}_{\alpha}(f)$. Since by assumption $\alpha$ is independent of $f(\mathbf{x}, t)$ the result applies to arbitrary distribution functions (including the Maxwellian case).

Let us now introduce the vector field $\mathbf{F}^{\prime}=\mathbf{F}+\Delta \mathbf{F}$, where the vector field $\Delta \mathbf{F}$ is assumed to depend functionally on $f(\mathbf{x}, t)$ and defined in such a way that:

A) the kinetic equation $L\left(\mathbf{F}^{\prime}\right) f(\mathbf{x}, t)=0$ yields an inverse kinetic theory for INSE, satisfying hypotheses 1-5 of the present theorem, and in particular it produces the same moment equation of the inverse kinetic equation (9) for $G(\mathbf{x}, t)=1, \mathbf{v}, E \equiv \frac{1}{3} u^{2} ;$

B) there results identically $\Delta \mathbf{F}\left(f_{M}\right) \equiv 0$, i.e., $\Delta \mathbf{F}$ vanishes identically in the case of a local Maxwellian distribution $f_{M}$.

Let us prove that necessarily $\Delta \mathbf{F}(f) \equiv 0$ also for arbitrary non-Maxwellian distributions $f$ which are solutions of the inverse kinetic equation. First we notice that from $\mathrm{A}$ and $\mathrm{B}$, due to hypotheses 3 and 4 , it follows that $\Delta \mathbf{F}$ must depend linearly on $\mathbf{Q}, \underline{\underline{\Pi}}-p_{1} \underline{\underline{\mathbf{1}}}$. On the other hand, again due to assumption A the vector field $\Delta \mathbf{F}$ must give a vanishing contribution to the moments the kinetic equation evaluated with respect to $G(\mathbf{x}, t)=1, \mathbf{v}, E \equiv \frac{1}{3} u^{2}$. Hence, in

order that also $\mathbf{F}^{\prime}$ depends only on the moments $\left\{\rho_{o}, \mathbf{V}, p_{1}, \mathbf{Q}, \underline{\underline{\Pi}}\right\}$ (hypothesis 4) necessarily it must result $\Delta \mathbf{F}(f) \equiv 0$ also for arbitrary non-Maxwellian distributions $f$. 


\section{Fulfillment of the energy equation}

As a further development, let us now impose the additional requirement that the inverse kinetic theory yields explicitly also the energy equation (8).

We intend to show that the kinetic equation fulfilling such a condition can be obtained by a unique modification of the mean-field force $\mathbf{F} \equiv \mathbf{F}_{0}(\mathbf{x}, t)+$

$\mathbf{F}_{1}(\mathbf{x}, t)$, in particular introducing a suitable new definition of the vector field $\mathbf{F}_{1}(\mathbf{x}, t)$ [Eq.(44), Appendix A]. The appropriate new representation is found to be

$$
\begin{aligned}
\mathbf{F}_{1}(\mathbf{x}, t ; f)=\frac{1}{2} \mathbf{u}\left\{\frac{\partial \ln p_{1}}{\partial t}-\frac{1}{p_{1}} \mathbf{V} \cdot\left[\frac{\partial}{\partial t} \mathbf{V}+\mathbf{V} \cdot \nabla \mathbf{V}+\right.\right. \\
\left.\quad+\frac{1}{\rho_{o}} \mathbf{f}-\nu \nabla^{2} \mathbf{V}\right]+ \\
\left.+\frac{1}{p_{1}} \nabla \cdot \mathbf{Q}-\frac{1}{p_{1}^{2}}[\nabla \cdot \underline{\underline{\Pi}}] \cdot \mathbf{Q}\right\}+ \\
+\frac{v_{t h}^{2}}{2 p_{1}} \nabla \cdot \underline{\underline{\Pi}}\left\{\frac{u^{2}}{v_{t h}^{2}}-\frac{3}{2}\right\} .
\end{aligned}
$$

As a consequence, the following result holds:

\section{THEOREM 2 - Inverse kinetic theory for extended INSE}

Let us require that:

1) assumptions 1-3 of Thm.1 are valid;

2) the mean-field $\mathbf{F}$ is defined:

$$
\mathbf{F}=\mathbf{F}_{0}+\mathbf{F}_{1}
$$

with $\mathbf{F}_{0}$ and $\mathbf{F}_{1}$ given by Eqs. (32) and (35).

Then it follows that:

A) $\{\rho, \mathbf{V}, p\}$ are classical solutions of extended INSE in $\Omega \times I$ [equations (1)-

(6) and (8)] if and only if the Maxwellian distribution function $f_{M}$ (10) is a particular solution of the inverse kinetic equation (9);

$B)$ provided that the solution $f(\mathbf{x}, t)$ of the inverse kinetic equation (9) exists in $\Gamma \times I$ and results suitably summable in the velocity space $U$, so that the moment equations of (9) corresponding to the weight-functions $G(\mathbf{x}, t)=1, \mathbf{v}, E \equiv \frac{1}{3} u^{2}$ exist, they coincide necessarily with extended INSE. 
C) the two representations (44) and (35) for $\mathbf{F}_{1}$ coincide identically

\section{PROOF:}

A) The proof is straightforward. In fact, recalling Thm.1 in [3] , we notice that Eqs. (35) and (44) manifestly coincide if and only if the energy equation (8) is satisfied identically, i.e., if the fluid fields are solutions of extended INSE.

B) The first two moment equations corresponding to $G(\mathbf{x}, t)=1, \mathbf{v}$ are manifestly independent of the form of $\mathbf{F}_{1}$, both in the case of Maxwellian and non-Maxwellian distributions, i.e., (35) and (44). Hence, in such a case Thm.3 of [3] applies, i.e., the moment equations yield INSE. Let us consider, in particular, the third moment equation corresponding to $G(\mathbf{x}, t)=\frac{1}{3} u^{2}$,

$$
\frac{\partial}{\partial t} p_{1}+\nabla \cdot \mathbf{Q}+\nabla \cdot\left[\mathbf{V} p_{1}\right]-\frac{2}{3} \int d \mathbf{v} \mathbf{F}(\mathbf{x}, t) \mathbf{u} f+\frac{2}{3} \nabla \mathbf{V}: \underline{\underline{\mathbf{\Pi}}}=0 .
$$

Invoking Eqs. (32) and (35) for $\mathbf{F}_{0}$ and $\mathbf{F}_{1}$, Eq.(14) reduces to

$$
p_{1} \nabla \cdot \mathbf{V}=0
$$

if and only if the energy equation (8) is satisfied. Since by construction $p_{1}>0$, this yields the isochoricity condition (3).

C) Finally, since thanks to A) $\{\rho, \mathbf{V}, p\}$ are necessarily classical solutions of INSE, it follows that they fulfill necessarily also the energy equation (8). Hence, (44) and (35) coincide identically in $\Gamma \times I$.

We conclude that (32) and (35) provide a new form of the inverse kinetic equation applying also to non-Maxwellian equilibria, which results alternative to that given earlier in [3]. The new form applies necessarily to classical solutions. Since weak solutions (and hence possibly also numerical solutions) of INSE may not satisfy exactly the energy equation, the present inverse kinetic theory based on the new definition given above [see Eq.(44)] for the vector field $\mathbf{F}(\mathbf{x}, t)$ provides a necessary condition for the existence of strong solutions of INSE. The result seems potentially relevant both from the conceptual viewpoint in mathematical research and for numerical applications.

\section{Conclusions}

In this paper the non-uniqueness of the definition of the inverse kinetic equation defined by Ellero and Tessarotto (see [3]) has been investigated, proving that the mean-field force $\mathbf{F}$ characterizing such an equation depends on an arbitrary real parameter $\alpha$. To resolve the indeterminacy, a suitably symmetrization condition has been introduced for the kinetic energy flux moment 
equation. As a consequence, the functional form the mean-field force $\mathbf{F}$ which characterizes the inverse kinetic equation results uniquely determined.

Finally, as an additional development, we have shown that, consistently with the assumption that the fluid fields are strong solutions of INSE, the meanfield force can be expressed in such a way to satisfy explicitly also the energy equation.

The result appears significant from the mathematical viewpoint, the physical interpretation of the theory and potential applications to the investigation of complex fluids, such as for example those treated in [5, 9]). In fact, it proves that the inverse kinetic theory developed in [3] can be given an unique form which applies to classical solutions of INSE.

ACKNOWLEDGEMENTS The research was developed in the framework of the PRIN Research Project "Modelli della teoria cinetica matematica nello studio dei sistemi complessi nelle scienze applicate" (Italian Ministry of University and Research). The authors are indebted with the reviewer for useful comments.

\section{Appendix A: relevant velocity moments and mean-field force}

Here we recall for completeness the expressions of the velocity-moments of the kinetic distribution function and of the mean-field force $\mathbf{F}$ given in [3]. The relevant moments are of the form

$$
M(G)=\int d^{3} v G(\mathbf{x}, t) f(\mathbf{x}, t)
$$

$G(\mathbf{x}, t)$ being the weight functions $G(\mathbf{x}, t)=1, \mathbf{v}, u^{2} / 3, \mathbf{u} u^{2} / 3, \mathbf{u} \mathbf{u}$. In particular, we identify the fluid fields $\left\{\rho \equiv \rho_{o}, \mathbf{V}, p\right\}$ with

$$
\begin{gathered}
\rho_{o}=\int d^{3} v f(\mathbf{x}, t), \\
\mathbf{V}(\mathbf{r}, t)=\frac{1}{\rho} \int d^{3} v \mathbf{v} f(\mathbf{x}, t), \\
p(\mathbf{r}, t)=p_{1}(\mathbf{r}, t)-P_{o},
\end{gathered}
$$

$P_{o}$ being a strictly positive real constant defined so that the physical realizability condition $p(\mathbf{r}, t) \geq 0$ is satisfied everywhere in the closure of the fluid domain $\bar{\Omega}$. Finally the expression of the mean-field force reads:

$$
\mathbf{F}(\mathbf{x}, t ; f)=\mathbf{F}_{0}(\mathbf{x}, t ; f)+\mathbf{F}_{1}(\mathbf{x}, t ; f)
$$


where $\mathbf{F}_{0}$ and $\mathbf{F}_{1}$ are the vector fields:

$$
\begin{gathered}
\mathbf{F}_{0}(\mathbf{x}, t ; f)=\frac{1}{\rho_{o}}\left[\nabla \cdot \underline{\underline{\Pi}}-\nabla p_{1}-\mathbf{f}\right]+\mathbf{u} \cdot \nabla \mathbf{V}+\nu \nabla^{2} \mathbf{V}, \\
\mathbf{F}_{1}(\mathbf{x}, t ; f)=\frac{1}{2} \mathbf{u}\left\{\frac{D}{D t} \ln p_{1}+\frac{1}{p_{1}} \nabla \cdot \mathbf{Q}-\frac{1}{p_{1}^{2}}[\nabla \cdot \underline{\underline{\Pi}}] \cdot \mathbf{Q}\right\}+ \\
+\frac{v_{t h}^{2}}{2 p_{1}} \nabla \cdot \underline{\underline{\Pi}}\left\{\frac{u^{2}}{v_{t h}^{2}}-\frac{3}{2}\right\},
\end{gathered}
$$

where the moments $p_{1}, \mathbf{Q}$ and $\underline{\boldsymbol{\Pi}}$ are given by Eqs.(18) - (21). In particular, for the Maxwellian kinetic equilibrium (10) there results identically

$$
\begin{aligned}
\underline{\underline{\Pi}} & =p_{1} \underline{\underline{\mathbf{1}}} \\
\mathbf{Q} & =\mathbf{0} .
\end{aligned}
$$

\section{Appendix B: Fokker-Planck representation}

It is interesting to point out that the choice of the parameter $\alpha=1 / 2$ and of the mean-field force $\mathbf{F}_{0}(\alpha)$ (32) can be obtained also by requiring that the Vlasov streaming operator (14) results "equivalent" to an appropriate FokkerPlanck operator (i.e., that it yields an inverse kinetic theory for INSE which admits local Maxwellian equilibria).

Since by assumption the parameter $\alpha$ does not depend functionally on $f(\mathbf{x}, t)$ it is sufficient to impose its validity only in the case of local Maxwellian equilibria $f_{M}$. In such a case, it is possible to require that there results identically

$$
L(\mathbf{F}) f_{M}=\left(\frac{\partial}{\partial t}+\mathbf{v} \cdot \frac{\partial}{\partial \mathbf{r}}\right) f_{M}+\sum_{i=1,2,3} \frac{\partial^{i}}{(\partial \mathbf{v})^{i}}\left\{\mathbf{D}_{i} f_{M}\right\} \equiv L_{F P}(\mathbf{F}) f_{M},
$$

where the Fokker-Planck coefficients $\mathbf{D}_{i}$ are assumed velocity-independent and $L_{F P}(\mathbf{F})$ denotes a Fokker-Planck operator which by construction is equivalent to the Vlasov operator $L(\mathbf{F})$. There results

$$
\begin{gathered}
\mathbf{D}_{1}\left(\mathbf{r}, t ; f_{M}\right)=-\frac{1}{\rho_{o}} \mathbf{f}+\nu \nabla^{2} \mathbf{V} \\
\underline{\underline{D}}_{2}\left(\mathbf{r}, t ; f_{M}\right)=-\frac{p_{1}}{\rho_{o}}\left\{\frac{1}{2} \nabla \mathbf{V}+\underline{\underline{\mathbf{I}}} \frac{1}{2}\left[\frac{\partial \ln p_{1}}{\partial t}+\mathbf{V} \cdot \nabla \ln p_{1}\right]\right\},
\end{gathered}
$$




$$
\underline{\underline{\underline{\mathrm{D}}}}_{3}\left(\mathbf{r}, t ; f_{M}\right)=\frac{1}{2} \underline{\underline{\underline{I}}}\left(\frac{p_{1}}{\rho_{o}}\right)^{2} \nabla \ln p_{1} .
$$

On the other hand, the relationship (47) holds if and only if

$$
\mathbf{F}(\alpha)=\mathbf{D}_{1}-\frac{\rho_{o}}{p_{1}}\left[\mathbf{u} \cdot \underline{\underline{\mathbf{D}}}_{2}+\underline{\underline{\mathbf{D}}}_{2} \cdot \mathbf{u}\right]-\left[2 \frac{\underline{\underline{1}}}{v_{t h}^{2}}+4 \frac{\mathbf{u u}}{v_{t h}^{2}}\right]: \underline{\underline{\underline{\mathbf{D}}}}_{3}(\mathbf{r}, t),
$$

namely the parameter $\alpha$ necessarily results equal to $1 / 2$, i.e.,

$$
\mathbf{F}_{0}\left(\alpha=\frac{1}{2}\right)=-\frac{1}{\rho_{o}} \mathbf{f}+\frac{1}{2}(\mathbf{u} \cdot \nabla \mathbf{V}+\nabla \mathbf{V} \cdot \mathbf{u})+\nu \nabla^{2} \mathbf{V} .
$$

\section{References}

[1] M. Ellero and M. Tessarotto, Bull. Am Phys. Soc. 45(9), 40 (2000).

[2] M. Tessarotto and M. Ellero, RGD24 (Italy, July 10-16, 2004), AIP Conf. Proceedings 762, 108 (2005).

[3] M. Ellero and M. Tessarotto, Physica A, doi:10.1016/ j.physa.2005.03.021 (2005).

[4] M. Tessarotto and M. Ellero, An H-theorem for incompressible fluids, submitted (2006); see also http://www.arxiv.org/physics/physics/0602136 (2006).

[5] M. Ellero, M. Kröger and S. Hess, Viscoelastic flows studied by Smoothed Particle Dynamics, J. Non-Newtonian Fluid Mech. 105, 35 (2002).

[6] H. Risken, The Fokker-Planck equation, Springer-Verlag, Berlin, 1984.

[7] R.F. Pawula, IEEE Trans. Inf.Th. 13, 1, 33 (1967).

[8] R.F. Pawula, Phys. Rev. 162, 186 (1967).

[9] M. Ellero, P. Español and E. G. Flekkoy, Phys. Rev. E 68, 041504 (2003).

[10] M. Ellero R.I. Tanner, SPH simulations of transient viscoelastic flows at low Reynolds number, submitted to J. Non-Newtonian Fluid Mech.(2005).

[11] I. Müller and T. Ruggeri, Rational Extended Thermodynamics (SpringerVerlag, Berlin, 1998). 